\title{
Desde las expectativas a la percepción de calidad de servicios en salud en Guayas, Ecuador
}

\author{
Miguel A. Bustamante ${ }^{(1,2) \star}$, Elsie Zerda ${ }^{(2)}$, Francisco Obando ${ }^{(3)}$ y Michelle Tello(2) \\ (1) Centro Interdisciplinario en Envejecimiento, Universidad de Talca. Dos Norte 685, 3465548 Talca, Chile. \\ (e-mail: mabu@utalca.cl) \\ (2) Facultad de Especialidades Empresariales, Universidad Católica de Santiago de Guayaquil, Av. Carlos Julio \\ Arosemena Km. 111/2 vía Daule, Guayaquil, Ecuador. (e-mail:elsie.zerda@cu.ucsg.edu.ec;michelle.tello@cu.ucsg.edu.ec) \\ (3) Facultad de Medicina, Universidad Católica de Santiago de Guayaquil, Av. Carlos Julio Arosemena Km. 11⁄2 vía \\ Daule, Guayaquil, Ecuador. (e-mail: francisco.obando@cu.ucsg.edu.ec)
}

Recibido May. 22, 2019; Aceptado Jul. 18, 2019; Versión final Ago. 8, 2019, Publicado Feb. 2020

\begin{abstract}
Resumen
Se comparan las expectativas con las percepciones de la calidad de servicio de salud a fin de determinar cuáles son las dimensiones que definen la calidad de servicio de los prestadores públicos de la salud primaria del Guayas, Ecuador. Se realizó un estudio cuantitativo, no experimental y transeccional con alcance descriptivo de una muestra de usuarios concurrentes a los servicios de atención primaria de salud. El muestreo fue de tipo probabilístico y se aplicó un cuestionario para la recogida de datos a una muestra de 533 individuos. Se aplicó estadística descriptiva y se verificó validez de contenido, validez convergente y confiabilidad a través del Alfa de Cronbach, y se realizó el análisis factorial exploratorio de expectativas y percepciones. Se concluye que los servicios generales prestados por los servicios de salud son valorados positivamente, con mayor frecuencia a favor de las mujeres. Se concluye también que los ítems que definen los factores de expectativas en general son comparables con los agentes que definen los factores de percepción.
\end{abstract}

Palabras clave: calidad de servicio; satisfacción usuaria; expectativas y percepciones de calidad; servicio de salud.

\section{From the expectations to the perception of quality of health services in Guayas, Ecuador}

\begin{abstract}
Expectations are compared with perceptions of the quality of health service to determine what are the dimensions that define the quality of service of public services of primary health in Guayas, Ecuador. A quantitative, non-experimental and transactional study was carried out with a descriptive scope of a sample of users attending the primary health care services. The sampling was probabilistic, and a questionnaire was applied to collect data from a sample of 533 people. Descriptive statistics were applied, and content validity, convergent validity and reliability were verified through Cronbach's Alpha, and an exploratory factor analysis of expectations and perceptions was performed. It is concluded that the general services provided by health services are positively valued, most often in favor of women. It is also concluded that the items that define the expectations factors in general, are similar to those reagents that define the perception factors.
\end{abstract}

Keywords: quality of service; user satisfaction; expectations and quality perceptions; health service 


\section{INTRODUCCIÓN}

La calidad se puede definir como un concepto relativo al uso esperado y percibido de un bien o servicio (Parasuraman, et al. 1988) que posee diferentes enfoques conceptuales (Fan et al., 2017). Algunos coinciden en que se refiere a la conjunción de rasgos y características de un producto o servicio, mientras que otros mencionan la capacidad relativa de éstos para satisfacer las necesidades y expectativas de un cliente (Adepoju et al., 2018). Progresivamente el concepto avanza al estudio del cumplimiento de las especificaciones definidas, mismas que se expresan en un conjunto de atributos apreciados por un tercero, sea este un cliente, un paciente o un usuario dispuesto a pagar por ellos (Fan et al., 2017). La calidad se entiende, entonces, como la mejor adecuación de un bien al uso así como a la percepción de satisfacción de las necesidades (Parasuraman et al., 1988). La persona, cliente, paciente o usuario percibe el bien o servicio como un eficaz satisfactor de sus necesidades (Adepoju et al., 2018) y dónde la calidad como apreciación subjetiva es apreciada desde un punto de vista determinado y singular (Fan et al., 2017).

En lo instrumental, se ha llegado a identificar aciertos y deficiencias en la calidad de servicio de las prestaciones en el contexto salubrista (Fan et al., 2017). Por una parte, definiendo la calidad en la atención médica como un concepto multidimensional que se basa en la percepción del paciente (Chasin y Galvi, 1998) y, por otra, definiendo que una atención médica de calidad dice relación con un tipo de atención que maximiza el bienestar del paciente abordando sistemáticamente dimensiones funcionales y técnicas significativas que se prestan con calidad (Galván et al., 2016). La calidad técnica refiere a la precisión de los análisis y técnicas médicas empleadas en el diagnóstico y diferentes fases de atención; mientras que la calidad funcional refiere a la forma en que se entregan dichos servicios (Adepoju et al., 2018). Así, la calidad técnica y la funcional resultan ser cruciales en los servicios de salud, ya que su evaluación permite mejorar el servicio rescatando sentimientos, expectativas y percepción efectiva por parte de los pacientes (Wysong y Driver, 2009).

En general, el concepto de servicio dice relación con acciones, procesos y ejecuciones intangibles, sin perjuicio que el soporte de los mismos sea tangible, dado que puede comprender hechos y acciones que se realizan para y con la participación de los usuarios (Zeithaml y Bitner, 2002). Estas prestaciones pueden ser valoradas dependiendo de la capacidad de percepción y apreciación de satisfacción experimentada por el perceptor. Un servicio congrega entonces un conjunto de prestaciones directas y complementarias asociadas a un bien intangible sostenido en un soporte tangible (Parasuraman et al., 1988), que en términos generales, se evidencia en acciones, procesos y servicios orientados al cumplimiento de unas especificaciones técnicas y funcionales que proporcionan satisfacción al usuario (Teshnizi et al., 2018).

El concepto de satisfacción es el resultado de la percepción íntima que experimenta un sujeto respecto de un servicio que alcanza y supera sus expectativas (Zeithaml y Bitner, 2002), en consecuencia, la satisfacción es un juicio acerca de los rasgos que caracterizan un producto o servicio por medio del cual se obtiene una percepción de saciedad, la cual genera en la persona un sentimiento de bienestar asociado a un momento placentero (Oliva e Hidalgo, 2004). La calidad, como apreciación subjetiva de valor, se traduce, en un conjunto de sensaciones de agrado que responden a las expectativas de un cliente (Kotler, 2001) mismas que se aprecian en un conjunto de satisfactores que conforman la calidad de servicio percibido (Oliva, 2004). Al respecto, Zeithalm y Bitner (2001). Es así como se identifican dos componentes fundamentales en esta percepción, a decir, el servicio deseado que determina las expectativas y el servicio adecuado que define lo pertinente en un determinado contexto, permitiendo al perceptor o cliente determinar lo que efectivamente estaría dispuesto a aceptar si los aprecia positivamente (Oliva e Hidalgo, 2004).

La percepción conceptualmente implica por una parte, un proceso de selección, organización e interpretación que realiza el cliente, paciente o usuario, respecto de un objeto que le reporta una cierta inteligibilidad del mundo que enfrenta (Galván et al., 2016), por otra parte, la percepción permite a la persona, en su rol de perceptor o receptor de un servicio, apreciar en sí mismo la sensación de satisfacción efectivamente experimentada (Zeithaml y Bitner,2002). Esta percepción a su vez puede ser medida por medio de constructos diseñados para el efecto, como es el propuesto por Parasuraman et al., (1988). En consecuencia, la calidad de servicio y la satisfacción del consumidor son conceptos diferenciados aunque claramente interdependientes (Freiberg et al., 2013) y por lo mismo se aplican conjuntamente en el ámbito de la salud, es por ello que la satisfacción usuaria se correlaciona con el cumplimiento de las expectativas del perceptor (Parasuraman et al., 1988) así como con un sentimiento de saciedad (Oliva e Hidalgo, 2004) asociado a un estado psicológico de satisfacción (Oliva e Hidalgo, 2004). En síntesis, se puede afirmar por lo tanto que la satisfacción es un hecho individual cuyo fundamento sustancial depende de la capacidad personal de apreciar la calidad de servicio efectivamente percibido y que es capaz de movilizar la conducta de un consumidor, cliente - usuario o paciente efectivamente satisfecho (Fan et al., 2017; Freiberg et al., 2013). Finalmente, sobre la base de los conceptos analizados, el presente trabajo compara las expectativas con las percepciones de la calidad de servicio a fin de determinar cuáles son las dimensiones que definen la calidad de servicio de los prestadores públicos de la salud primaria del Guayas, Ecuador. 


\section{METODOLOGÍA}

El estudio adopta un enfoque cuantitativo (Chen et al., 2008) no experimental y transeccional (Forero y Gómez, 2017) con alcance descriptivo (Hernández et al., 2016) de una muestra de usuarios concurrentes a los servicios de atención primaria de salud, APS del Guayas, Ecuador. Se trabajó en la ciudad de Guayaquil por ser la más poblada de Ecuador (INEC, 2014) considerando además que a nivel nacional se registraron 43.268.343 consultas de morbilidad, prevención, ginecobstetricia y emergencia, de las cuales 27.586 .691 se dieron en centros APS equivalente a un $63,75 \%$, aportando datos que, trasladados a la provincia de Guayas, alcanza a 8.192.632 pacientes atendidos a nivel de APS, equivalente a un $28,74 \%$ de las consultas a nivel nacional.

\section{Población y muestra}

La población en estudio quedó constituida por los habitantes de la ciudad de Guayaquil, misma que, de acuerdo con el Instituto Nacional de Censo, cuenta con 2.350.915 habitantes (INEC, 2014), de los cuales 1.473.336 tienen edades entre 18 a 64 años, con lo cual se excluyen de la muestra los menores de edad (s18). El muestreo fue de tipo probabilístico considerando un nivel de confianza de $95 \%$, varianza máxima (50\%) y un error tolerable de $5 \%$, obteniéndose una muestra $\geq 500$ individuos, considerada buena (Lloret et. al. 2014) lo que supera el criterio de muestra mínima $n \geq 300$ (Lloret et al. 2014) y el criterio de personas por ítem (N/p) considerando las reglas de 10:1 y 5:1 que determina el número de casos necesarios por cada uno de los reactivos del instrumento (Lloret et al., 2014), en consecuencia, dado que el instrumento cuenta con 22 reactivos, la muestra permite superar los criterios analizados.

\section{Instrumento}

El instrumento de recogida de datos fue un cuestionario (Hernández et al., 2016) de 22 reactivos tomados de Parasuraman et al., (1985; 1988) debidamente validados (Cohen et al., 2008) que congrega reactivos en cinco dimensiones latentes que se denominan Fiabilidad, Seguridad, Elementos tangibles, Capacidad de respuesta y Empatía, todas factibles de ser medidos en valores organizados en escala Likert de 1 - 7 puntos unidireccionales crecientes (Freiberg et al., 2013; Pruzan, 2016). Las cinco dimensiones y sus 22 reactivos del cuestionario son evaluadas primero, en término de las expectativas, relativas a las predicciones hechas por los consumidores sobre lo que es probable que suceda durante la transacción o servicio, representando los deseos del consumidor acerca del servicio que se ofrece y segundo, en término de las percepciones que recogen el grado de satisfacción que efectivamente las personas aprecian del producto o servicio recibido. En consecuencia, mediante la comparación de expectativas y percepciones de los reactivos y dimensiones valoradas en escala de Likert, es posible determinar el grado de coincidencia o discrepancia que se puede producir entre las expectativas iniciales del consumidor respecto de las percepciones finales de satisfacción (Parasuraman et al., 1988). Sin perjuicio del origen, validez y confiabilidad del instrumento, el cuestionario fue sin embargo validado para confirmar palabras, expresiones y lenguaje mediante consulta con un grupo de expertos provenientes del sector APS del Guayas y piloteado con una sub muestra, asegurando que la misma no forme parte de la muestra final del estudio (Hernández et al., 2016).

\section{Recolección de datos}

La recolección de datos fue llevada a cabo entre los meses de junio y agosto del año 2018 con la participación de encuestadores debidamente entrenados (Hernández et al., 2016) quienes abarcaron los cinco centros de salud ubicados en el norte y sur de la ciudad (INEC, 2010). Los centros médicos participantes fueron el Dispensario de Distrito 09D09 Tarqui, Puerto Hondo, Chongón, Estrella de Belén y Papa Francisco en Samanes y Guasmo (INEC, 2010). Los encuestadores lograron alrededor de 50 cuestionarios cada semana, totalizando al cabo de la segunda semana de agosto, 582 cuestionarios los cuales fueron depurados. Para ello se revisaron inconsistencias y completitud de los registros, optándose por eliminar aquellos con ítems vacíos y respuesta iguales en todos los ítems, entre otros. Una vez depurada la base de datos, se obtuvo un total de 533 cuestionarios válidos (Lloret et al., 2014).

\section{Técnicas de análisis de datos, validez y confiabilidad}

El análisis de datos se inició con la estadística descriptiva (Hernández et al., 2016) aplicada al análisis de los datos sociodemográficos de la muestra y a la percepción de los servicios generales entregados por los prestadores de los servicios APS. Por otra parte, en la medición de los reactivos, se verificó normalidad de los datos, asimetría y curtosis, además de los índices de Kolomorov-Smirnov a nivel univariante (Urrutia et al., 2014; Pérez y Medrano, 2010) y test de Mardia para el análisis multivariante (Gao et al., 2008).

Seguidamente se procedió a ratificar la validez de contenido (Hernández et al., 2016; Urrutia et al., 2014) analizando el instrumento sobre la base de la teoría propuesta por Parasuraman et al., (1988). La validez 
convergente se analizó mediante análisis factorial de los reactivos verificando la conformación de dimensiones representativas (Freiberg et al., 2013; Lloret, et al., 2014), previa verificación del índice KMO y la prueba de esfericidad de Barltett que comprueba la pertinencia de los datos para el análisis factorial (Freiberg et al., 2013). La confiabilidad se determinó a través del Alfa de Cronbach (Cohen et al., 2002; Freiberg et al., 2013) para comprobar consistencia interna de los datos por medio del análisis del promedio de las correlaciones entre los ítems, que permite comprobar la coherencia de los reactivos (Johnson y Wichern, 2009) y ratificarlos de acuerdo con los criterios de Ruiz (1998).

\section{Análisis factorial}

Se aplicó el análisis factorial exploratorio AFE a fin de sintetizar los 22 reactivos de expectativas y de percepciones (Kline, 2011; Pérez y Medrano, 2010) conformando factores convergentes y mutuamente comparables (Hair et al., 2017; Pérez, et al., 2000), lo que fue ratificado mediante la varianza explicada de cada factor y los acumulados de expectativas y percepciones, la cual se obtuvo utilizando rotación varimax (Lloret, et al., 2014) e independencia entre los factores (Freiberg et al., 2013). Finalmente, se optó por retener para el análisis los reactivos persistentes (Méndez y Rondón, 2012) considerando los índices de comunalidad y cargas factorial más altas $(\geq 0,5)$.

\section{RESULTADOS}

Se presentan los resultados y hallazgos de expectativas y precepciones de calidad de servicio, comenzando con la caracterización de la muestra, luego con los servicios generales, siguiendo con las expectativas y percepciones de los ítems de calidad de servicio, terminando con el análisis comparativo de factores de expectativas y percepciones.

\section{Caracterización de la muestra}

Dentro de la caracterización de la muestra que se presenta en la tabla 1, se evidencia que, una mayoría de participantes son de género femenino, quienes representan más de las tres cuartas de la muestra. Así mismo, la mayoría de los individuos encuestados se ubican entre las edades de 21 a 45 años representando el $80 \%$. La mayor parte de la muestra vive en pareja reportando unión libre o casado. La escolaridad de los entrevistados muestra que más del $50 \%$ tiene un nivel de secundaria completa y, respecto del empleo, un porcentaje importante reportó encontrarse desempleado y de aquellos con empleo la mayoría corresponde a trabajadores informales.

Tabla 1: Características socio demográficas de la muestra

\begin{tabular}{|c|c|c|c|c|c|c|c|c|}
\hline Variable & $n$ & $\%$ & Variable & $n$ & $\%$ & Variable & $n$ & $\%$ \\
\hline Género & & & Nivel de escolaridad & & & Situación laboral & & \\
\hline Masculino & 108 & 20,3 & Primaria incompleta & 17 & 3,2 & Desempleado & 131 & 24,6 \\
\hline Femenino & 412 & 77,3 & Primaria completa & 23 & 4,3 & Jubilado & 3 & 0,6 \\
\hline Edad & & & Secundaria incompleta & 121 & 22,7 & $\begin{array}{l}\text { Trabajos menores ocasionales } \\
\text { e informales }\end{array}$ & 161 & 30,2 \\
\hline Menos de 20 años & 26 & 4,9 & Secundaria completa & 286 & 53,7 & $\begin{array}{l}\text { Oficio menor, obrero no } \\
\text { calificado, peón }\end{array}$ & 51 & 9,6 \\
\hline 21 a 25 años & 130 & 24,4 & Técnico Tecnológico & 20 & 3,8 & Servicio doméstico con contrato & 10 & 1,9 \\
\hline 26 a 30 años & 192 & 36 & $\begin{array}{l}\text { Universidad } \\
\text { incompleta }\end{array}$ & 34 & 6,4 & $\begin{array}{l}\text { Obrero calificado, albañil, } \\
\text { operador maquinista, capataz }\end{array}$ & 35 & 6,6 \\
\hline 31 a 45 años & 131 & 24,6 & Universidad completa & 20 & 3,8 & Micro empresario & 66 & 12,4 \\
\hline 46 a 60 años & 39 & 7,3 & Posgrado & 1 & 0,2 & Empleado administrativo & 37 & 6,9 \\
\hline Más de 61 años & 10 & 1,9 & & & & $\begin{array}{l}\text { Gerente empresa pequeña, } \\
\text { profesional }\end{array}$ & 9 & 1,7 \\
\hline & & & & & & $\begin{array}{l}\text { Gerente empresa grande, } \\
\text { profesional con prestigio }\end{array}$ & 1 & 0,2 \\
\hline
\end{tabular}

\section{Percepción de los servicios generales}

Las percepciones de los servicios generales recogidas en escala de siete alternativas, fue analizada a nivel descriptivo determinando las frecuencias de percepción de hombres y mujeres, quienes concentraron sus respectivas respuestas en las dos últimas opciones que indican Satisfecho y Totalmente satisfecho. Las frecuencias de los hombres se ubicaron por encima de los 90 casos, en tanto que las mujeres alcanzaron frecuencias por encima de los 370 , cuatro veces por encima de los hombres, a causa de ser éstas las que, por lo general, concurren a los servicios de atención primaria de salud, como se detalla en la tabla 2. 
De la tabla 2 se observa así mismo que los servicios generales de salud se congregaron en un solo factor perfectamente determinado con una varianza explicada alta de $60,246 \%$ significativa al $1 \%$ y con tres componentes altamente correlacionados marcando cargas factoriales por encima de los 0,85 puntos, considerando previamente la medida de adecuación muestral de Kaiser-Meyer-Olkin KMO de 0,725 y la prueba de esfericidad de Bartlett que determina un Chi-cuadrado aproximado de 765,404, con 6 grados de libertad y un nivel de significancia alto al $1 \%(0,000)$. Complementariamente, en lo que respecta a los ítems, las percepciones de los usuarios se ubicaron en los rangos más altos entre las opciones satisfechos y muy satisfechos, para la afirmación: los trámites necesarios para ser atendido fueron adecuados, para el ítem, la calidad de atención médica recibida en su última visita fue alta, terminando con el reactivo, los tiempos de espera para ser atendido por el profesional médico, que resultaron en general satisfactorios.

Tabla 2: Percepciones de calidad de los servicios generales en salud

\begin{tabular}{|c|c|c|c|c|c|c|c|c|}
\hline \multirow{2}{*}{ Reactivos } & & & & & & \multicolumn{3}{|c|}{ Frecuencia } \\
\hline & & & & & Factor 1 & Homk & & Mujer \\
\hline \multicolumn{5}{|c|}{$\begin{array}{l}\text { Respecto a los asuntos de trámites para ser atendido en la unidad médica, usted } \\
\text { se encuentra }\end{array}$} & 0,905 & \multicolumn{2}{|c|}{91} & 388 \\
\hline \multicolumn{5}{|c|}{$\begin{array}{l}\text { En términos generales, respecto a la calidad de atención médica recibida en su } \\
\text { última visita a la unidad médica, usted se encuentra }\end{array}$} & 0,854 & \multicolumn{2}{|c|}{97} & 389 \\
\hline \multicolumn{5}{|c|}{$\begin{array}{l}\text { Respecto a los tiempos de espera para ser atendido por el profesional médico, } \\
\text { usted se encuentra }\end{array}$} & 0,852 & \multicolumn{2}{|c|}{92} & 373 \\
\hline \multicolumn{5}{|c|}{ Varianza explicada } & $60,246 \%$ & & & \\
\hline \multirow{3}{*}{$\begin{array}{l}\text { Factor } \\
\text { Servicios } \\
\text { Generales }\end{array}$} & \multicolumn{3}{|c|}{ Autovalores iniciales } & \multicolumn{5}{|c|}{$\begin{array}{c}\text { Sumas de las saturaciones al cuadrado de la } \\
\text { extracción }\end{array}$} \\
\hline & Total & $\%$ de la varianza & $\%$ acumulado & Total & \multicolumn{2}{|c|}{$\%$ de la varianza } & \multicolumn{2}{|c|}{$\%$ acumulado } \\
\hline & 2,410 & 60,246 & 60,246 & 2,410 & \multicolumn{2}{|c|}{60,246} & & 60,246 \\
\hline
\end{tabular}

\section{Expectativas y percepciones de los ítems de calidad de servicio}

En general la tabla 3 muestra los resultados del análisis de los 22 reactivos del modelo de Parasuraman et al., (1985; 1988). Por una parte, se observa que las expectativas de calidad de servicio se agruparon en tres factores alcanzando una varianza explicada de 63,6\%, que alcanzan un índice de Kaiser-Meyer-Olkin, KMO de 0,933 y una prueba de esfericidad de Bartlett que entregó un Chi-cuadrado aproximado de 8560,124, con 231 grados de libertad, significativo al $1 \%(0,000)$, confirmando 21 reactivos y dejando sin carga factorial significativa el componente El profesional médico debería inspirar confianza al paciente. Por otra parte, las percepciones dieron forma a cuatro factores que explican un $61,971 \%$ de la varianza total, previa determinación del índice de Kaiser-Meyer-Olkin, KMO de 0,923 y una prueba de esfericidad de Bartlett que entregó un Chi-cuadrado aproximado de 5429,370, con 190 grados de libertad, significativo al 1\% $(0,000)$, ratificando 21 ítems, quedando con carga factorial insuficiente y fuera del análisis el componente El profesional médico debería mostrar interés para solucionar su problema de salud. Así mismo se observa, en términos agregados, que las expectativas promedio en escala 1 - 7 puntos se ubican en 6,75 para el ítem La consulta con el profesional médico de la sala de atención debería realizarse en horario y fecha determinada y 6,9149 puntos para el componente El profesional médico debería ser cortés (amable), estableciendo un rango de expectativas que, en promedio, alcanza 6,8834 puntos y que finalmente se expresa en una distancia media entre expectativas y percepciones que llega a 0,1822 puntos equivalente a un $2,6 \%$ de desviación.

Un análisis más detallado de los reactivos (tabla 3) agrupados en tres factores de expectativas y cuatro factores de percepciones, presenta algunas convergencias y divergencias significativas que ahora se analizan. Se observa que el factor 1 de expectativas denominado Calidad técnica y funcional, con 11 componentes $(26,571 \%)$ es altamente coincidente con el primer factor de percepciones denominado Calidad técnica y atención personalizada con 9 ítems (24,684\%) coincidiendo en 8 reactivos, en consecuencia, este factor efectivamente permite contrastar ambas apreciaciones ratificando los hallazgos con cierta certeza. El segundo factor de expectativas llamado Comunicación y oportunidad de atención con cinco ítems (20,702\%) se confronta con el factor 3 de percepciones que representa el Acceso a servicio y funcionalidad de la atención y que contiene cuatro reactivos $(18,684 \%)$, coincidiendo solo en tres de ellos, lo cual demuestra que estos factores son claramente divergentes.

El factor tres de expectativas llamado Atención personalizada y acceso a servicio con cinco ítems (16,327\%) entregó resultados significativos al ser contrastado con los demás factores de percepciones, determinándose que se contrasta perfectamente con el segundo factor de percepciones denominado Comunicación y oportunidad de atención, que contiene los mismos reactivos $(13,681 \%)$, confirmando una perfecta convergencia de estos factores y la pertinencia de medida de los reactivos asociados. 
Tabla 3: Factores de expectativa y percepción de calidad de servicio

\begin{tabular}{|c|c|c|c|c|c|c|c|c|c|c|}
\hline & \multicolumn{4}{|c|}{ Factores de expectativa } & \multicolumn{5}{|c|}{ Factores de percepción } & \multirow{2}{*}{$\begin{array}{c}\text { Distancia } \\
\text { media }\end{array}$} \\
\hline & 1 & 2 & 3 & Prom. & 1 & 2 & 3 & 4 & Prom. & \\
\hline $\begin{array}{l}\text { La sala de consulta debería estar limpia, } \\
\text { cómoda y acogedora }\end{array}$ & 0,803 & & & 6,888 & 0,641 & & & & 6,770 & 0,118 \\
\hline $\begin{array}{l}\text { La sala de consulta debería contar con } \\
\text { materiales necesarios para el diagnóstico } \\
\text { (guantes, estetoscopio, termómetro, etc.) }\end{array}$ & 0,773 & & & 6,897 & 0,654 & & & & 6,775 & 0,122 \\
\hline $\begin{array}{l}\text { El profesional médico debería tener una } \\
\text { presentación personal adecuada, usar } \\
\text { uniforme que lo identifica }\end{array}$ & 0,724 & & & 6,912 & 0,694 & & & & 6,827 & 0,085 \\
\hline $\begin{array}{l}\text { El profesional médico debería siempre estar } \\
\text { dispuesto a ayudarle }\end{array}$ & 0,688 & & & 6,912 & 0,606 & & & & 6,810 & 0,102 \\
\hline \multirow{3}{*}{$\begin{array}{l}\text { El profesional médico nunca debería estar } \\
\text { demasiado ocupado para responder sus } \\
\text { preguntas }\end{array}$} & 0,665 & & & 6,878 & & & & & 6,930 & $-0,052$ \\
\hline & \multicolumn{4}{|c|}{ Factores de expectativa } & \multicolumn{5}{|c|}{ Factores de percepción } & \multirow{2}{*}{$\begin{array}{c}\text { Distancia } \\
\text { media }\end{array}$} \\
\hline & 1 & 2 & 3 & Prom. & 1 & 2 & 3 & 4 & Prom. & \\
\hline $\begin{array}{l}\text { Usted debería sentirse seguro con el } \\
\text { tratamiento brindado por el profesional médico }\end{array}$ & 0,650 & & & 6,902 & 0,705 & & & & 6,726 & 0,176 \\
\hline $\begin{array}{l}\text { El profesional médico debería responder de } \\
\text { manera segura a sus preguntas e inquietudes } \\
\text { en relación a su enfermedad }\end{array}$ & 0,645 & & & 6,902 & 0,725 & & & & 6,752 & 0,150 \\
\hline $\begin{array}{l}\text { El profesional médico debería atenderlo a la } \\
\text { hora que le corresponde }\end{array}$ & 0,642 & & & 6,891 & & & 0,551 & & 6,654 & 0,237 \\
\hline $\begin{array}{l}\text { La sala de consulta debería contar con } \\
\text { equipamiento necesario, moderno y óptimo } \\
\text { para realizar un buen tratamiento }\end{array}$ & 0,603 & & & 6,867 & & & & 0,745 & 6,649 & 0,218 \\
\hline $\begin{array}{l}\text { El profesional médico debería ser cortés } \\
\text { (amable) }\end{array}$ & 0,599 & & & 6,915 & 0,731 & & & & 6,735 & 0,180 \\
\hline $\begin{array}{l}\text { El profesional médico debería comunicarle } \\
\text { con exactitud su cita y próximo control }\end{array}$ & 0,560 & & & 6,893 & 0,647 & & & & 6,777 & 0,116 \\
\hline $\begin{array}{l}\text { El profesional médico debería inspirar } \\
\text { confianza al paciente }\end{array}$ & & & & 7,006 & 0,606 & & & & 6,701 & 0,305 \\
\hline \begin{tabular}{|l|} 
El profesional médico debería ser capaz de \\
comprender claramente lo que usted necesita
\end{tabular} & & 0,787 & & 6,889 & & & & & 6,806 & 0,083 \\
\hline $\begin{array}{l}\text { El profesional médico debería escucharlo } \\
\text { atentamente y tratarlo con amabilidad, respeto } \\
\text { y paciencia }\end{array}$ & & 0,779 & & 6,915 & & & 0,511 & & 6,820 & 0,095 \\
\hline $\begin{array}{l}\text { El profesional médico debería mostrar interés } \\
\text { para solucionar su problema de salud }\end{array}$ & & 0,753 & & 6,893 & & & & & 6,833 & 0,060 \\
\hline $\begin{array}{l}\text { La sala de consulta médica debería tener } \\
\text { horarios de trabajo convenientes para la } \\
\text { atención a los usuarios }\end{array}$ & & 0,661 & & 6,893 & & & 0,770 & & 6,765 & 0,128 \\
\hline $\begin{array}{l}\text { La sala de consulta médica debería ofrecer } \\
\text { atención individualizada }\end{array}$ & & 0,608 & & 6,897 & & & 0,771 & & 6,731 & 0,166 \\
\hline $\begin{array}{l}\text { El profesional médico debería explicarle de } \\
\text { forma ordenada y clara en qué consiste los } \\
\text { procedimientos de atención }\end{array}$ & & & 0,847 & 6,790 & & 0,768 & & & 6,483 & 0,307 \\
\hline $\begin{array}{l}\text { La consulta con el profesional médico de la } \\
\text { sala de atención debería realizarse en horario } \\
\text { y fecha determinada }\end{array}$ & & & 0,783 & 6,754 & & 0,778 & & & 6,302 & 0,452 \\
\hline $\begin{array}{l}\text { En el centro médico el doctor o enfermera } \\
\text { debería dar a conocer los procedimientos que } \\
\text { se realizarán durante el proceso de } \\
\text { diagnóstico }\end{array}$ & & & 0,698 & 6,878 & & 0,640 & & & 6,604 & 0,274 \\
\hline $\begin{array}{l}\text { El centro médico debería entregar un buen } \\
\text { servicio desde la primera vez que usted acude } \\
\text { como paciente }\end{array}$ & & & 0,655 & 6,835 & & 0,784 & & & 6,493 & 0,342 \\
\hline $\begin{array}{l}\text { En el centro médico las horas disponibles } \\
\text { existentes deberían ser fáciles de obtener } \\
\text { para la cita con el doctor }\end{array}$ & & & 0,532 & 6,829 & & 0,555 & & & 6,482 & 0,347 \\
\hline Promedios & & & & 6,883 & & & & & 6,701 & 0,182 \\
\hline Varianza explicada por el factor & 26,571 & 20,702 & 16,327 & & 24,684 & 18,064 & 13,681 & 5,542 & & \\
\hline Varianza total explicada & & & & 63,6 & & & & & 61,971 & \\
\hline
\end{tabular}


Finalmente, el factor 4 de percepciones, denominado Equipamiento e instalaciones, cuenta con tan solo un componente referido a las condiciones materiales de La sala de consulta debería contar con equipamiento necesario, moderno y óptimo para realizar un buen tratamiento, que sin perjuicio de su relevancia intrínseca, resulta insuficiente para definir un factor (Lloret, et al., 2014) y en consecuencia, debe ser técnicamente excluido del análisis (Hair et al., 2017).

\section{Análisis comparativo de factores de expectativas y percepciones}

De la tabla 4 se determina que, si el factor 1 incrementa su comportamiento, induce incrementos directos y en el mismo sentido de los restantes dos factores, sobre F2 en 0,569 y F3 en 0,464. Por otra parte, si el factor 2 incrementa, se generan decrementos e incrementos en los restantes factores, sobre $F 1$ en $-0,120$ y sobre F3 en 0,835 . Por último si el factor 3 incrementa su resultado, incide en forma inversa y en forma directa sobre los demás factores, sobre F1 en -0,725 y sobre F2 en 0,622.

Tabla 4: Comparativo de las varianzas totales explicadas y matrices de transformación de los factores

\begin{tabular}{|c|c|c|c|c|c|c|c|c|c|c|}
\hline \multicolumn{11}{|c|}{ Varianza total explicada expectativas } \\
\hline \multirow[t]{2}{*}{ Componente } & \multicolumn{4}{|c|}{ Autovalores iniciales } & \multicolumn{3}{|c|}{$\begin{array}{c}\text { Sumas de las saturaciones al } \\
\text { cuadrado de la extracción }\end{array}$} & \multicolumn{3}{|c|}{$\begin{array}{c}\text { Suma de las saturaciones al } \\
\text { cuadrado de la rotación }\end{array}$} \\
\hline & Total & $\begin{array}{c}\% \text { de la } \\
\text { varianza }\end{array}$ & \multicolumn{2}{|c|}{$\%$ acumulado } & Total & $\begin{array}{c}\% \text { de la } \\
\text { varianza }\end{array}$ & $\%$ acumulado & Total & \begin{tabular}{c|c}
$\%$ de la \\
varianza
\end{tabular} & $\%$ acumulado \\
\hline 1 & 11,312 & 51,418 & \multicolumn{2}{|c|}{51,418} & 11,312 & 51,418 & 51,418 & 5,846 & 26,571 & 26,571 \\
\hline 2 & 1,512 & 6,873 & \multicolumn{2}{|c|}{58,291} & 1,512 & 6,873 & 58,291 & 4,554 & 20,702 & 47,273 \\
\hline 3 & 1,168 & 5,310 & \multicolumn{2}{|c|}{63,600} & 1,168 & 5,310 & 63,600 & 3,592 & 16,327 & 63,600 \\
\hline \multicolumn{11}{|c|}{ Varianza total explicada percepciones } \\
\hline \multicolumn{11}{|l|}{ Componente } \\
\hline 1 & 8,675 & 43,376 & 43,376 & \multicolumn{2}{|c|}{8,675} & 43,376 & 43,376 & 4,937 & 24,684 & 24,684 \\
\hline 2 & 1,569 & 7,846 & 51,223 & \multicolumn{2}{|c|}{1,569} & 7,846 & 51,223 & 3,613 & 18,064 & 42,748 \\
\hline 3 & 1,096 & 5,480 & 56,703 & \multicolumn{2}{|c|}{1,096} & 5,480 & 56,703 & 2,736 & 13,681 & 56,429 \\
\hline 4 & 1,054 & 5,268 & 61,971 & & 054 & 5,268 & 61,971 & 1,108 & 5,542 & 61,971 \\
\hline Matriz d & transform & ación de la & s expectati & vas & & Matriz & iz de transforma & ción de I & las percepcic & iones \\
\hline Componentes & 1 & 2 & 2 & 3 & & 1 & 2 & 3 & & 4 \\
\hline 1 & 0,679 & 0,5 & 569 & 0,46 & & 0,705 & 0,537 & 0,45 & & 0,070 \\
\hline 2 & $-0,120$ & $-0,5$ & 537 & 0,83 & & 0,391 & $-0,833$ & 0,34 & & 0,176 \\
\hline 3 & $-0,725$ & 0,6 & 22 & 0,29 & & $-0,591$ & 0,088 & 0,77 & & 0,190 \\
\hline 4 & & & & & & $-0,006$ & 0,095 & $-0,25$ & & 0,963 \\
\hline
\end{tabular}

Del análisis de las percepciones se determinan cuatro factores que conforman un sistema parcialmente determinado. El factor 1, se correlaciona en forma directa (positiva) con los demás factores sobre F2 en 0,705, sobre F3 en 0,537 y sobre F4 en 0,457. El segundo factor por su parte, incide en forma directa e inversa sobre los demás factores, sobre $\mathrm{F} 1$ en 0,391 , sobre $\mathrm{F} 3$ en 0,349 y sobre $\mathrm{F} 4$ en 0,176 , en tanto que el factor 3 , incide inversamente en uno de los factores y directamente en los demás, sobre F1 en , -0,591, sobre F2 en 0,088y sobre F4 en 0,190 .

Por último, el cuarto factor, explica un 5,542\% de la varianza total quedando conformado por un solo reactivo, con una carga factorial alta de 0,745 pero que sin embargo resulta insuficiente para sostener, por sí mismo, el análisis. Este último factor, como se observa en la tabla 5, presenta correlaciones bajas con los demás factores, sobre F1, F2 y F3; en -0,006, 0,095 y -0,251, respectivamente, en consecuencia, este factor resulta desestimado del análisis.

\section{DISCUSIÓN}

Las percepciones de los servicios generales de hombres y mujeres se ubicaron entre las opciones satisfecho y totalmente satisfecho, evidenciando una apreciación conjunta de alto valor equivalente a un $86,3 \%$ del total entrevistado con lo que se hacen evidentes unas ideas asociadas al concepto de calidad (Zun et al., 2018) las cuales son claramente percibidas por los usuarios - pacientes (Galván et al., 2016), por supuesto, con mayor frecuencia entre las mujeres que entre los hombres (370 / 90), como habitualmente ocurre cuando se prestan servicios de salud de cobertura familiar en APS (Oliva e Hidalgo, 2004)

El análisis factorial de los servicios generales concentró los reactivos en un solo factor, confirmando la convergencia requerida (Pérez y Medrano, 2010) marcando un 60,246\% de varianza explicada, al $1 \%$ y con tres componentes con cargas factoriales por encima de los 0,85 puntos. De esta forma se verifica la validez de constructo de las percepciones de los servicios generales (Pérez et al., 2000; Urrutia et al., 2014) dando 
pie a un hallazgo de investigación interpretable. En consecuencia, las percepciones de los usuarios parecen valorar positivamente los servicios generales que se les entregan en APS (Teshnizi et al., 2018) y que en este trabajo se traducen en la adecuación de los trámites necesarios para ser atendido y, por supuesto, en la calidad de la atención médica recibida (Zun et al., 2018), apreciando tiempos adecuados y pertinentes de atención (Wysong y Driver, 2009).

Del análisis de las expectativas se determinaron tres factores con reactivos que explican el $63,6 \%$ de la varianza total (Méndez et al., 2012; Pérez y Medrano, 2010), dejando fuera del análisis el componente El profesional médico debería inspirar confianza al paciente, lo que puede ser explicado, porque este ítem es ya un componente de servicio logrado (Zeithaml et al., 2002) y, por lo mismo, es parte consustancial de la excelencia asumida por los usuarios (Chen et al., 2008) y, por lo mismo, se da como hecho realizado y siempre disponible para los pacientes (Adepoju et al., 2018).

Por otra parte, las percepciones se congregaron en cuatro factores (Freiberg et al., 2013) que explican un $61,971 \%$ de la varianza, pero que sin embargo, excluye uno de los reactivos analizados, a decir: El profesional médico debería mostrar interés para solucionar su problema de salud, exclusión que puede deberse al hecho que, aunque existe una idea de lo que significa calidad en los servicios de salud (Galván et al. 2016), esto no resulta claramente apreciado por los usuarios (Jandavath y Byram, 2016), lo cual puede ocurrir tanto a nivel de la salud pública como a nivel de la salud privada (Javed y llyas, 2018).

Al analizar las coincidencias entre los factores, el primer factor de expectativas resultó claramente coincidente con el primer factor de percepciones, identificando componentes que pueden ser denominados de excelencia (Chen et al., 2008). Esto se aprecia en los 8 reactivos coincidentes y que por lo mismo, por una parte deberían ser la expresión de un desempeño exitoso en salud (Forero y Gómez, 2017; Zun et al., 2018) y que, por otra, debería permitir ratificar la apreciación de la calidad del servicio percibido en APS (Adepoju et al., 2018), en tanto sean los servicios debidamente apreciados y definidos en lo que socialmente es la idea de la calidad de un servicio (Galván, et al., 2016) y que naturalmente se asocia a un desempeño de excelencia en las prestaciones (Teshnizi et al., 2018), en este caso, de los servicios de salud. El segundo factor de expectativas por su parte, resultó parcialmente concordante con el tercer factor de percepciones (Lloret et al., 2014), coincidiendo en cuatro reactivos lo cual confirma (Adepoju et al., 2018), con un cierto grado de divergencia, la adecuación que se produce entre estos dos factores (Méndez et al., 2012).

Por último, uno de los principales hallazgos de la investigación indican que en las expectativas, el factor tres con cinco ítems se compara directamente con el segundo factor de percepciones (Chen et al., 2008) coincidiendo en todos sus reactivos (Freiberg et al., 2013), lo cual ratifica que estos dos factores recogen adecuadamente un conjunto de reactivos de expectativas finalmente coincidentes que son confirmados como significativos por las percepciones de los usuarios (Forero y Gómez, 2017). En general, los tres primeros factores de expectativas de la calidad de servicios (Fan et al. 2017) presentan correlaciones altas $(\geq 0,4)$ ubicándose por encima del exigido $(\geq 0,3)$ por los estudios factoriales (Freiberg et al. 2013; Hair et al. 2017) y, por lo mismo, conforman un sistema de factores (Méndez et al., 2012; Pérez y Medrano, 2010) debidamente consistente (Kline, 2011; Lloret et al., 2014; Zun et al., 2018) que permiten determinar, de manera agregada, cuales son los factores y sus respectivos reactivos que definen la calidad de servicio de la atención de salud primaria entregado por los prestadores APS del Guayas, Ecuador.

\section{CONCLUSIONES}

El análisis de los resultados de este trabajo permite concluir que los servicios generales prestados por los servicios de salud del Guayas, tales como los trámites necesarios para ser atendido, la calidad de atención médica recibida y los tiempos de espera para ser atendido, son valorados positivamente tanto por los hombres como por las mujeres, con mayor frecuencia a favor de las mujeres, confirmando este contenido como pertinente para definir un concepto de calidad de servicio en un solo factor efectivamente apreciado por los usuarios o pacientes.

Se concluye así mismo que los ítems que definen los factores de expectativas se contrastan a la par con los reactivos que definen los factores de percepción, determinándose que las expectativas de la calidad de servicio se agruparon en tres factores congruentes y las percepciones de la calidad dieron forma a tres factores relevantes parcialmente congruentes con los primeros, dónde destaca la coincidencia del factor tres de expectativas con cinco ítems se contrasta perfectamente con el segundo factor de percepciones en todos sus reactivos, lo cual ratifica que estos dos factores recogen adecuadamente los ítems de las expectativas debidamente percibidas como satisfactorias por los usuarios de los servicios de salud del Guayas, Ecuador. 
En atención a que las dimensiones de las expectativas son parcialmente concordantes con los de percepción, se pudieron identificar cuáles son los componentes de calidad de servicio de excelencia que se identifican como relevante denominándose en consecuencia los factores calidad técnica y funcional, comunicación y oportunidad de atención y atención personalizada y acceso a servicio, evidenciando de esta forma un sistema de reactivos que definen, en opinión de los usuarios, lo que debe ser llamado un desempeño exitoso, por parte de los prestadores de servicios de salud a nivel de APS que efectivamente respondan a las necesidades y que son percibidos satisfactorios por los usuarios o pacientes del Guayas, Ecuador.

\section{REFERENCIAS}

Adepoju, O. O., Z. Opafunso y M. Ajayi, Primary Health Care in South West Nigeria: Evaluating service quality and patients' satisfaction. African Journal of Science, Technology, Innovation and Development, 10(1), 13-19 (2018)

Chen, Y., J. Spohrer y A. Lelescu, Three factors to sustainable service system excellence: A case study of service systems. IEEE, International Conference on Services Computing (2008)

Cohen, L., L. Manion y K. Morrison, Research methods in education. London and New York, Routledge (2002)

Fan, L. H., L. Gao, X. Liu, S. H. Zhao, H. T. Mu, Z. Li y F. G. Lou, Patients' perceptions of service quality in China: An investigation using the SERVQUAL model. PloS one, 12(12), e0190123 (2017)

Forero, D. E., y A. Gómez, Comparison of measurement models based on expectations and perceived performance for the satisfaction study in health services. suma psicológica, 24(2), 87-96 (2017)

Freiberg, H. A., J. B. Stover, G. de la Iglesia, M. Fernández, Polychoric And Tetrachoric Correlations In Exploratory And Confirmatory Factorial Studies. Prensa Médica Latinoamericana - ISSN 1688-4094 Ciencias Psicológicas, VII (2): 151 $164(2013)$

Galván, H. R., J. M. Moctezuma, G. F. Dolci y D. O. López, De la idea al concepto en la calidad en los servicios de salud. Revista Conamed, 17(4) (2016)

Gao, S., P. Mokhtarian y R. Johnston, Nonnormality of data in structural equation models. Transportation Research Record: Journal of the Transportation Research Board, 20(82), 116-124 (2008)

Hair, J.F., G.T.M. Hult, C. Ringle y M. Sarstedt, A primer on partial least squares structural equation modeling (PLS-SEM). Sage Publications (2017)

Hernández Sampieri Roberto, Carlos Fernández Collado, Pilar Baptista Lucio, Metodología De La Investigación. Cuarta edición. Editorial Mc Graw Hill año 2016 pág. 435 (2016)

Instituto Nacional de Estadísticas y Censo Anuario de Estadística de Salud: Recursos y Actividades 2014 (2014)

Instituto Nacional de Estadísticas y Censo Censo Nacional. Infografía Ciudad de Guayaquil (2010)

Jandavath, R. K. N. y A. Byram, Healthcare service quality effect on patient satisfaction and behavioural intentions in corporate hospitals in India. International Journal of Pharmaceutical and Healthcare Marketing, 10(1), 48-74 (2016)

Javed, S. A., S. Liu, A. Mahmoudi y M. Nawaz, Patients' satisfaction and public and private sectors' health care service quality in Pakistan: Application of grey decision analysis approaches. The International journal of health planning and management;1-15 (2018)

Johnson, R. y D. Wichern, Applied Multivariate Statistical Analysis (5th ed., pp 1-767). New Jersey: Prentice-Hall International (2009)

Kline, R.B. Principles and practice of structural equation modelling. (3rd. Ed.). New York: Guilford Press (2011)

Kotler, Philip, Gary Armstrong Marketing. 8º Edición. Mexico: Prentice Hall (2001)

Lloret, S., A. Ferreres, A. Hernández y I. Tomás, El análisis factorial exploratorio de los ítems: una guía práctica, revisada y actualizada. Anales de psicología, 30(3), 1151-1169 (2014)

Méndez Martínez, C. y M. A. Rondón Sepúlveda, Introducción al análisis factorial exploratorio. Revista colombiana de psiquiatría, 41(1), 197-207 (2012)

Oliva, C. y C. Hidalgo, Satisfacción Usuaria: Un Indicador De Calidad Del Modelo De Salud Familiar, Evaluada En Un Programa De Atención De Familias En Riesgo Biopsicosocial, En La Atención Primaria. Psykhe, Nov. 2004, Vol.13, No.2, P.173 $186(2004)$

Parasuraman, A., V. A. Zeithaml y L. L. Berry, Servqual: A multiple-item scale for measuring consumer perc. Journal of retailing, 64(1), 12 (1988)

Pérez, E. R. y L. A. Medrano, Análisis factorial exploratorio: bases conceptuales y metodológicas. Revista Argentina de Ciencias del Comportamiento (RACC), 2(1), 58-66 (2010)

Pérez, J. A., S. C. Moscoso y R. M. Rodríguez, Validez de constructo: el uso de análisis factorial exploratorio-confirmatorio para obtener evidencias de validez. Psicothema, 12(Suplemento), 442-446 (2000) 
Pruzan, P. Research Methodology: The Aims, Practices and Ethics of Science . Cham: Springer. DOI: 10.1007/978-3-31927167-5 (2016)

Razali, N. M. y Y. B. Wah, Power comparisons of shapiro-wilk, kolmogorov-smirnov, lilliefors and anderson-darling tests. Journal of statistical modeling and analytics, 2(1), 21-33 (2011)

Teshnizi, S. H., T. Aghamolaei, K. Kahnouji, S. M. H. Teshnizi y J. Ghani, Assessing quality of health services with the SERVQUAL model in Iran. A systematic review and meta-analysis. International Journal for Quality in Health Care, 30(2), 82-89 (2018)

Urrutia, M., S. Barrios, M. Gutiérrez, Métodos óptimos para determinar validez de contenido. Educación Médica Superior, 28(3), 547-558 (2014)

Wysong, P. R. y E. Driver, Patients' perceptions of nurses' skill. Critical Care Nurse, 29(4), $24-37$ (2009)

Zeithaml, Valerie y Mary Jo Bitner, Marketing de Servicios: Un enfoque de integración del cliente a la empresa. México, DF: McGraw Hill (2002)

Zun, A. B., M. I. Ibrahim y A. A. Hamid, Level of Satisfaction on Service Quality Dimensions Based on SERVQUAL Model Among Patients Attending 1 Malaysia Clinic in Kota Bharu, Malaysia. Oman medical journal, 33(5), 416-422 (2018) 